\title{
Fluvoxamina no transtorno depressivo maior: um estudo multicêntrico aberto
}

\author{
Fluvoxamine in the treatment of major depressive \\ disorder: an open multicentric study
}

José Alberto Del Porto', Andréa Feijó de Mello, Florence Kerr-Correa², Andrés dos Santos Jr. ${ }^{3}$, Ricardo Alberto Moreno ${ }^{4}$, Carlos Henrique Rodrigues dos Santos 5 , Aline Valente Chaves ${ }^{4}$, Marcio Versiani ${ }^{5}$, Antônio Egídio Nardiं, Irismar Reis de Oliveira ${ }^{6}$, Mônica Gonçalves Ribeiro ${ }^{6}$, Flávio Kapczinski ${ }^{7}$, Fernando Gazalle? Benício Frey’, Sérgio Tamai ${ }^{8}$

\section{RESUMO}

Objetivo: Este trabalho estudou a eficácia e a tolerabilidade da fluvoxamina no tratamento, de forma aberta, sem comparação com placebo ou outros agentes, por 6 semanas, de pacientes com o diagnóstico de transtorno depressivo maior (TDM). Constitui-se em objetivo secundário do estudo avaliar os efeitos da fluvoxamina sobre o sono dos pacientes. Métodos: Foram incluídos 104 pacientes, maiores de 18 anos, com o diagnóstico de TDM, de acordo com os critérios do Manual Diagnóstico e Estatístico de Transtornos Mentais, 4a edição (DSM-IV), e com escores, na Escala de Hamilton para Depressão, versão de 17 itens (HAM-D 17), de 17 pontos ou mais. Avaliou-se a eficácia da fluvoxamina por meio das Escalas HAM-D 17 e da CGI (Impressão Clínica Global). A análise dos itens 4, 5 e 6 da HAM-D 17 foi utilizada para a avaliação do sono dos pacientes. Avaliaram-se a segurança e a tolerabilidade da fluvoxamina ao longo das 6 semanas, registrando-se quaisquer eventos adversos. A fluvoxamina foi inicialmente ministrada em doses de 50 ou $100 \mathrm{mg} / \mathrm{dia}$, podendo haver aumentos progressivos até 300 mg/dia. Resultados: Dos 104 pacientes incluídos, 81 (78\%) concluíram o estudo. Obtiveram resposta favorável (diminuição de 50\% ou mais na HAM-D 17) 69\% dos pacientes, e a taxa de remissão (HAM-D $17 \leq 7$ ) foi de 52\%. A análise da CGI indicou ter havido melhora significante ( $p<0,001)$ em relação aos escores de base. A análise específica dos itens relativos ao sono, na HAM-D 17, revelou melhora significativa já na segunda visita, mantendo-se ao longo das 6 semanas. Os eventos adversos foram os esperados para inibidores seletivos de recaptação da serotonina, predominando as queixas gastrointestinais, em sua maioria transitórias e de pequena intensidade. Conclusão: $O$ estudo vem confirmar a eficácia e a tolerabilidade da fluvoxamina no tratamento do transtorno depressivo maior, assim como sua eficácia no tratamento das alterações do sono encontradas nos pacientes deprimidos. O perfil de eventos adversos foi o esperado para os ISRS, ressaltando-se o fato de que poucos pacientes relataram disfunção sexual (2,5\% dos pacientes).

1 Universidade Federal de São Paulo (Unifesp).

2 Hospital das Clínicas da Faculdade de Medicina de Botucatu da Universidade Estadual Paulista (Unesp).

3 Hospital do Servidor Público Estadual de São Paulo (HSPE-SP).

4 Hospital das Clínicas da Faculdade de Medicina da Universidade de São Paulo (FMUSP).

Recebido

13/12/2006

Aprovado

5 Instituto de Psiquiatria da Universidade Federal do Rio de Janeiro (UFRJ).

6 Casa de Saúde Ana Nery.

7 Hospital das Clínicas da Faculdade de Medicina da Universidade Federal do Rio Grande do Sul (UFRGS).

8 Faculdade de Ciências Médicas da Santa Casa de São Paulo. 


\section{ABSTRACT}

Objective: This research studied the efficacy and tolerability of fluvoxamine in the treatment of major depressive disorder (MDD), during 6 weeks, in an open trial, without placebo or active comparator. A secondary objective was the evaluation of the effects of fluvoxamine on the sleep of the pacients. Methods: 104 patients were inicially included, with the diagnosis of MDD in accordance to the criteria of the Diagnostic and Statistical Manual for Mental Disorders, $4^{\text {th }}$ edition (DSM-IV). Patients should have scores $\geq 17$ in the Hamilton Depression Scale for Depression 17 itens (HAM-D 17). The efficacy of fluvoxamine was studied through the HAM-D 17 and CGI (Clinical Global Impression). The analysis of the HAM-D 17 itens 4, 5, and 6 was used for the evaluation of the quality of sleep of the patients. Security and tolerability of fluvoxamine was assessed throughout the six weeks, being registered any adverse event. Fluvoxamine was inicially administered at the doses of 50 or $100 \mathrm{mg} /$ day; the doses could be progressively increased until 300 mg/day. Results: From the 104 included patients, 81 (78\%) concluded the study. Sixty nine percent (69\%) of the patients obtained favorable response (defined as 50\% improvement in the HAM-D 17) and the remission rate (HAM-D $17 \leq 7)$ was 52\%. The specific analysis of CG/ showed significant improvement $(p<0.001)$ comparing to the baseline scores. The speficic analysis of the sleep itens of the HAM-D 17 showed significant improvement from the $2^{\text {nd }}$ week; the improvement was sustained until the end of the 6 weeks study. The adverse events were those expected for the serotonin selective reuptake inhibitors (SSRI), predominantly gastrointestinal complaints, transitory and of low intensity in most of the cases. Conclusion: This study confirms the efficacy and tolerability of fluvoxamine in the treatment of MDD, and also its efficacy in the treatment of sleep disturbs among depressed patients. The profile of adverse events were those expected for SSRI. It should be emphasized that few patients reported sexual disfunction (2.5\% of the patients).

\section{INTRODUÇÃO}

O papel da serotonina na gênese dos estados depressivos foi primeiramente postulado há cerca de 40 anos (Lapin e Oxenkrug, 1969). Desde essa época, inúmeros estudos com modelos animais de depressão, estudos clínicos com pacientes deprimidos, estudos com depleção de serotonina e mesmo estudos post-mortem têm dado suporte a essa hipótese (Curzon, 1982; Delgado et al., 1999).

A eficácia dos ISRS, para o tratamento dos transtornos depressivos, encontra-se bem estabelecida, assim como para alguns estados de ansiedade. Em relação aos antidepressivos tricíclicos, os ISRS mostram-se mais seguros quanto ao perfil de efeitos colaterais, em particular quanto à cardiotoxicidade. Caracterizam-se também pela relativa falta de efeitos anticolinérgicos, sendo também menos sedativos que os tricíclicos (Montgomery, 1994).

A fluvoxamina, um ISRS cujos primeiros estudos referentes às depressões remontam ao início da década de 1980 (Feldman e Denber, 1982), tem se mostrado eficaz mesmo no tratamento das depressões severas. Assim, a fluvoxamina foi utilizada com sucesso no tratamento de depressões graves, com escores na HAM-D $\geq 25$ (Ottevanger, 1991). A fluvoxamina foi comparada à clomipramina, mostrando-se ambas igualmente eficazes no tratamento das depressões graves (Zohar et al., 2003). A fluvoxamina foi também utilizada no tratamento das depressões delirantes (Gatti et al., 1996; Zanardi et al., 1998); sua eficácia em monoterapia nas depressões psicóticas tem sido atribuída a sua alta afinidade pelos receptores sigma de tipo $1\left(\delta_{1}\right)$, de acordo com Stahl (2005).

Tem-se amplamente utilizado a fluvoxamina também no tratamento dos transtornos de ansiedade, entre eles o transtorno obsessivo-compulsivo (Mundo et al., 2001), a ansiedade generalizada, a fobia social, o transtorno de estresse pós-traumático e os estados mistos de ansiedade e depressão (Laws et al., 1990), além da depressão maior com ansiedade comórbida (Sonawalla et al., 1999).

Em que pesem os numerosos trabalhos existentes a respeito da eficácia e segurança da fluvoxamina, ainda não existem (até onde vai o nosso conhecimento) ensaios clínicos realizados no Brasil, com esse medicamento, o que vem justificar o presente trabalho.

\section{MÉTODOS}

O presente estudo foi realizado em oito centros de investigação no Brasil (ver nota no final do texto), visando a estu- 
dar a eficácia e a segurança da fluvoxamina no tratamento, por 6 semanas, de pacientes com transtorno depressivo maior (TDM), definida pelos critérios do DSM-IV (1994). O estudo foi realizado de forma aberta, sem comparação com placebo ou outros medicamentos.

No decorrer de aproximadamente 5 meses, entre outubro de 2004 e março de 2005, foram arrolados 104 pacientes maiores de 18 anos com o diagnóstico de transtorno depressivo maior (DSM-IV) e escores na Escala de Hamilton para Depressão, versão de 17 itens (HAM-D 17), acima ou igual a 17 pontos.

O protocolo foi aprovado pelos Comitês de Ética e Pesquisa (CEP) de cada centro participante do estudo, tendo os pacientes preenchido o termo de consentimento livre e esclarecido (TCLE), sendo-lhes assegurado, a qualquer momento, o direito de sair do estudo e de dar continuidade ao tratamento de forma habitual nos serviços em que eram regularmente atendidos. Os critérios de exclusão foram os que se seguem: hipersensibilidade à medicação, insuficiência hepática ou renal, gravidez ou amamentação e uso de inibidores da monoaminoxidase nas 2 semanas anteriores ao estudo. Foram excluídos também pacientes com depressão bipolar (transtorno bipolar do humor) ou condições comórbidas, como esquizofrenia, transtorno esquizofreniforme, transtornos delirantes e transtornos psicóticos sem outra especificação, assim como pacientes com risco de suicídio. Durante o período do estudo, os pacientes não fizeram uso de outros antidepressivos, antipsicóticos, anticonvulsivantes, anticoagulantes, cisaprida, teofilina, ciclosporina, metoprolol, terfenadina e astemizol.

O objetivo primário do estudo foi avaliar a eficácia da fluvoxamina no tratamento de pacientes com transtorno depressivo maior, utilizando-se as Escalas HAM-D 17 e CGl (Impressão Clínica Global). Na primeira visita, além da história clínica, exame físico e dados demográficos, foram registrados o peso dos pacientes e o uso de eventuais medicações concomitantes. Em cada visita, os pacientes foram novamente submetidos a exame físico habitual e foram avaliados quanto a quaisquer eventos adversos; também se registrou o uso de quaisquer medicações concomitantes. Ao final do estudo, os pacientes tiveram novamente seu peso reavaliado. A HAM-D 17 foi aplicada na visita 1 e nos dias 7, 21, 28 e 42; a CGI foi aplicada na visita 1 e ao fim da 6a semana (dia 42). Constituiu-se em objetivo secundário a avaliação da qualidade do sono dos pacientes, avaliada por meio dos itens 4, 5 e 6 da HAM-D 17. A segurança e a tolerabilidade da fluvoxamina foram também avaliadas ao longo das 6 semanas, registrando-se quaisquer eventos adversos. Os pacientes foram avaliados, quanto aos eventuais efeitos colaterais, no período basal (dia 0) e, a seguir, nos dias 7, 21, 28 e 42 após o início da medicação. A dose inicial foi de 50 ou $100 \mathrm{mg}$ de fluvoxamina, administrada à noite. As doses poderiam ser reajustadas em $50 \mathrm{mg}$ a cada semana, até o máximo de 300 mg ao dia. Doses acima de 150 mg/dia foram administradas em duas tomadas.

\section{Análise dos dados}

Para a análise da evolução da Escala de Hamilton ao longo das visitas, fez-se uso da análise de variância para mensurações repetidas. Na análise final, utilizou-se a última observação carreada até o final (last observation carried forward). A análise dos itens da Escala de Hamilton relativos ao sono (itens 4, 5 e 6) foi feita por meio do Teste de Friedman. A análise da CGI (Impressão Clínica Global) foi realizada empregando-se o Teste de Wilcoxon.

\section{RESULTADOS}

Iniciaram o estudo 104 pacientes, sendo 81 (77,9\%) do sexo masculino e $23(22,1 \%)$ do feminino. A idade média foi de 45 anos (desvio-padrão de 12,6 anos), variando entre 19 e 75 anos.

Dos 104 pacientes que iniciaram o estudo, 81 (78\%) concluíram o seguimento previsto, e 60 (58\%) continuaram com a medicação após o término do estudo. Dos 23 pacientes que não concluíram o estudo, apenas dois o fizeram por indicação médica (efeitos adversos) e dois por alegada falta de eficácia. Seis pacientes não retornaram para dar seqüência ao tratamento (perda de acompanhamento), e um deixou de se tratar por razões administrativas. Doze pacientes pediram para sair do estudo em virtude de supostos eventos adversos, sem que houvesse indicação médica para sua interrupção.

As doses empregadas variaram entre 50 e $300 \mathrm{mg} / \mathrm{dia}$. A maioria dos pacientes (42/81) que terminou o estudo finalizou-o em uso de $100 \mathrm{mg} /$ dia (51,9\% dos pacientes). Na visita final, 1,2\% dos pacientes estavam em uso de $50 \mathrm{mg} / \mathrm{dia}, 51,9 \%$, em uso de $100 \mathrm{mg} / \mathrm{dia}, 22,2 \%$, em uso de $150 \mathrm{mg} / \mathrm{dia}$, 18,5\%, em uso de $200 \mathrm{mg} / \mathrm{dia}$ e 6,2\%, em uso de $300 \mathrm{mg} / \mathrm{dia}$.

A evolução dos pacientes, quanto aos escores da Escala de Hamilton de 17 itens (HAM-D), foi submetida à análise de variância para mensurações repetidas, levando-se em conta apenas aqueles pacientes que concluíram o estudo $(n=81)$.

Os dados mostram que, nas sucessivas visitas, houve diminuição dos escores na HAM-D. Assim, observou-se diferença estatisticamente significante já ao fim da 1ạ semana de tratamento $(p<0,001)$. A análise de variância para medidas repetidas mostra diferenças estatisticamente significantes $(p<0,001)$ na média dos escores de cada visita. Essa diferença se expressa por meio de uma tendência (quadrática) de queda ao longo das visitas, que começa já ao fim da 1a semana (segunda visita) e tende a diminuir entre as duas últimas visitas (em função de já ter havido diminuição acentuada dos escores da HAM-D). 
A evolução dos pacientes, quanto aos escores da HAM$D$, pode ser vista na figura 1 .

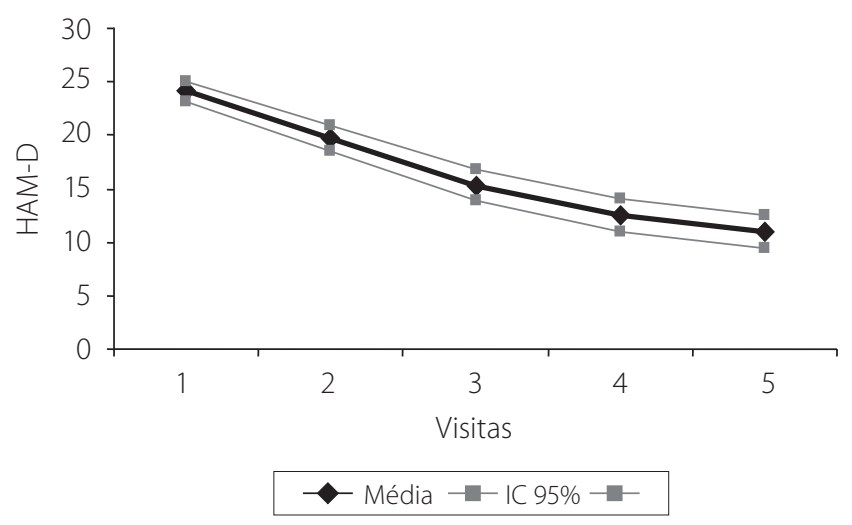

Figura 1. Evolução dos escores totais da Escala de Hamilton (HAM-D) e IC 95\%.

A figura 2 mostra a evolução dos escores totais da HAM-D, utilizando-se o método da LOCF (Last Observation (Carried Forward), que leva em conta a última avaliação daqueles que, por qualquer motivo, abandonaram o tratamento. Os escores da última observação realizada são tratados como se fossem obtidos ao fim do tratamento.

\section{Evolução do escore total da escala de Hamilton (LOCF),} IC 95\%, Luvox, 2005

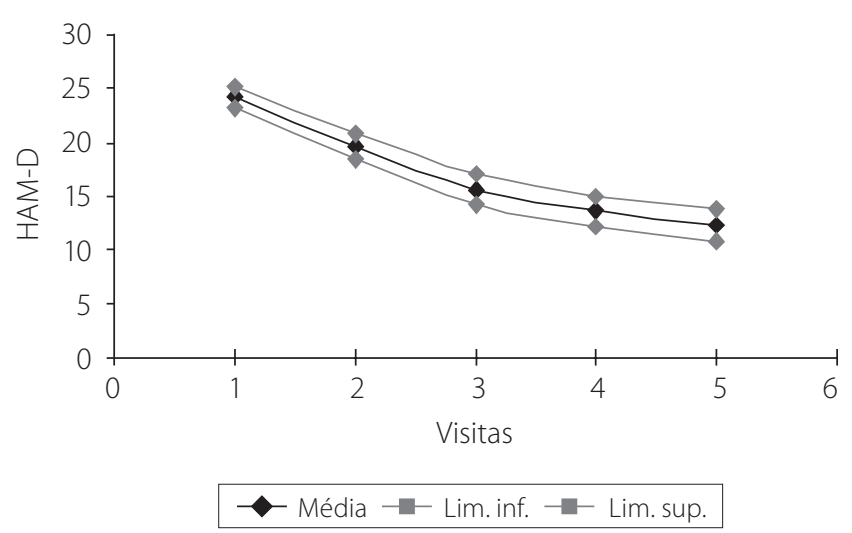

Figura 2. Evolução dos escores totais da HAM-D 17, utilizando-se o método da LOCF.

O estudo daqueles que obtiveram resposta e remissão foi feito mediante a análise de sobrevida (survival analysis).

A porcentagem daqueles que tiveram resposta (diminuição de, no mínimo, 50\% dos escores na HAM-D) foi de 69\% (IC 95\%: 54-74\%) e daqueles que obtiveram remissão (HAM-D $\leq 7$ ) foi de 52\% (IC 95\%: 39-65\%), como se pode ver na figura 3.

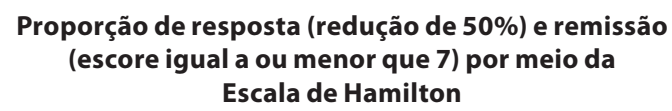

$\%$

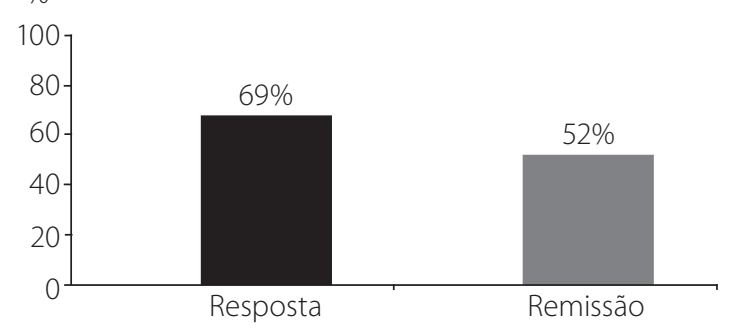

Figura 3. Taxas de resposta (redução $\geq 50 \%$ ) e remissão $(\leq 7)$ de acordo com os escores na HAM-D 17 ao fim do estudo.

A análise dos itens da Escala de Hamilton relativos às alterações do sono (itens 4, 5 e 6), por meio do Teste de Friedman, revelou melhora significante já na segunda visita ( $p<0,001)$, melhora que se manteve ao longo das 6 semanas de avaliação. A análise individualizada de cada item (itens 4, 5 e 6) mostrou também melhora estatisticamente significante, de acordo com o Teste de Friedman (Item 4: Qui-Quadrado = 47,2; $4 \mathrm{gl}$; $\mathrm{p}<0,001$; item 5: Qui-Quadrado= 34,5; 4 gl; item 6: Qui-Quadrado= 52,6; 4 gl, p < 0,001).

O Teste de Wilcoxon indicou ter havido melhora significativa no que diz respeito à $C G I(p<0,001)$. Os escores médios da CGI, ao início do estudo, foram de 4,6 (dp =0,8) e os escores finais, de 2,7 ( $d p=1,5)$.

Não houve diferença no peso dos pacientes entre a primeira e a última visita $(p>0,25)$.

Os pacientes apresentaram boa adesão ao tratamento. Dos 104 pacientes que iniciaram o estudo, 81 (78\%) o completaram.

O número de pacientes que relataram efeitos adversos foi de $65,7 \%$ na visita 2 e de apenas $16 \%$ na visita final, mostrando que os eventos adversos foram transitórios e melhoraram com a continuidade do tratamento e o ajuste das doses. Os eventos colaterais com possível ou provável relação com a fluvoxamina e que afetaram 2,5\% ou mais dos pacientes foram: náuseas (22,7\%), cefaléia (10,3\%) tonturas $(9,9 \%)$, sonolência $(7,4 \%)$, dor de estômago $(3,4 \%)$, insônia (3,9\%), diarréia (3\%) e diminuição da libido (2,5\%).

O número de pacientes que fazia uso de medicações concomitantes caiu de 39,4\% na primeira visita para 9,9\% na última, diferença que se mostrou estatisticamente significante, de acordo com o Teste de McNemar $(p<0,005)$.

Alguns pacientes já faziam uso de benzodiazepínicos, como hipnóticos, ao iniciarem o estudo (clonazepam, $\mathrm{n}=8$; zoldipem, $n=7$; lorazepam, $n=6$; flunitrazepam, $n=2$; alprazolam, $n=2$; bromazepam, $n=1$ ). Outras medicações utilizadas incluíram: diuréticos, $n=7$ (hidroclorotiazida, clortalidona, furosemida); analgésicos, $n=7$ (paracetamol, dipirona, ácido acetilsalicílico); betabloqueadores, $n=5$ (atenolol, propranolol); captopril e enalapril, $n=5$; omeprazol e pantoprazol, $n=4$. 


\section{DISCUSSÃO}

Os resultados deste estudo sugerem ser a fluvoxamina eficaz e segura no tratamento da depressão maior. Os dados alinham-se em conformidade a numerosos estudos realizados em outros centros (Feighner et al., 1989; Nemeroff et al., 1995; Ottevanger, 1991; Haffmans et al., 1996; Zanardi et al., 2000) que vêm demonstrar sua utilidade na terapêutica dos estados depressivos.

A mediana da dose empregada ao fim do estudo foi de 100 mg, dose coincidente com aquela (102 mg, dp = 35 mg) encontrada no estudo de Rapaport et al. (1996).

O escore médio inicial, na HAM-D de 17 itens, foi de 24,1 pontos ( $d p=5,3$ ), sendo o final de 10,9 pontos $(d p=7,0$ ), diferença estatisticamente significante $(p<0,001)$.

Confirmando estudos prévios, mostrou-se também útil a fluvoxamina em reduzir os escores relativos a alterações do sono (itens 4, 5 e 6 da HAM-D) já a partir da 1a semana do tratamento. Cumpre notar que, já ao final da 1a semana de tratamento, observou-se diminuição estatisticamente significante dos escores da HAM-D ( $p<0,001)$. As taxas de resposta ao tratamento (diminuição de $50 \%$ ou mais dos escores na HAM-D) foram de $69 \%$ ao fim da 6 a semana, taxas equivalentes àquelas encontradas no estudo de Dalery e Honig (2003), que foram de aproximadamente $60 \%$. As taxas de resposta encontradas neste estudo foram também similares às taxas encontradas por Zohar et al. (2003), para pacientes com depressão severa (HAM-D $\geq 25$ ), que foram de $71 \%$; este último estudo, no entanto, teve duração mais longa, de 8 semanas.

Neste estudo, a taxa de remissão (HAM-D $\leq 7)$ foi de $52 \%$ ao final da $6^{a}$ semana. Para fins de comparação, Thase et al. (2005) realizaram uma metanálise de sete estudos controlados com placebo, encontrando taxas de remissão, na 8 a semana de tratamento, de $47 \%$ para pacientes tratados com ISRS (inibidores seletivos de recaptação de serotonina) e de $36 \%$ para o placebo. Deve-se ressalvar que, em estudos abertos, as taxas de remissão são geralmente maiores que em estudos duplo-cegos, em que os pacientes e os avaliadores têm ciência de que uma parte do grupo estará recebendo placebo.

Não houve diferença no peso dos pacientes entre a primeira e a última visita $(p>0,25)$. Ao contrário de outros antidepressivos (como os tricíclicos e a mirtazapina), que induzem a considerável ganho de peso, a fluvoxamina, segundo alguns autores, tem sido até recomendada para o tratamento do comer compulsivo (Appolinario e McElroy, 2004). Esse dado alinha-se em conformidade a estudos realizados com animais de laboratório, que atribuem a inibição do apetite (em ratos) à ação da fluvoxamina sobre os receptores $5 \mathrm{HT}_{1 \mathrm{~B}}$ (Nonogaki et al., 2006).

O perfil de eventos colaterais foi o esperado para os inibidores seletivos de recaptação da serotonina, predominando os eventos gastrointestinais (náuseas, dor de estô- mago e diarréia). Outros eventos foram: cefaléia, tonturas, sonolência, insônia e diminuição da libido. Os eventos relativos à disfunção sexual foram de pequena monta (2,5\% dos pacientes). Embora esses efeitos não tenham sido pesquisados de forma sistemática por meio de questionário específico neste estudo, é importante ressaltar que a baixa ocorrência de efeitos colaterais, no que tange à atividade sexual, tem sido apontada pela literatura corrente (Westenberg e Sandner, 2006; Buchberger e Wagner, 2002).

Os eventos adversos foram, em sua maioria, transitórios e de pequena intensidade, diminuindo a partir da $2^{\underline{a}}$ semana. Cumpre ressaltar que apenas 2 entre os 104 pacientes $(1,9 \%)$ interromperam o estudo por orientação dos médicos, em virtude de eventos colaterais.

Este trabalho resultou de um estudo observacional, tendo as limitações inerentes a um ensaio clínico aberto. Os estudos abertos estão sujeitos às influências das expectativas tanto dos pacientes quanto dos investigadores envolvidos. A ausência de um grupo placebo implica a impossibilidade do controle dos fatores inespecíficos que podem contribuir para a melhora clínica dos pacientes.

Por outro lado, tratando-se de um estudo naturalístico, seus resultados tendem a se aproximar mais daqueles obtidos na prática habitual, refletindo a realidade clínica do cotidiano.

\section{CONCLUSÃO}

Os resultados desse estudo sugerem a eficácia e a boa tolerabilidade da fluvoxamina no transtorno depressivo maior, assim como sua utilidade quanto ao tratamento das alterações do sono encontradas em pacientes deprimidos.

Nota: Participaram da pesquisa os seguintes centros: Universidade Federal de São Paulo (Unifesp, São Paulo), Hospital das Clínicas da Faculdade de Medicina de Botucatu da Universidade Estadual Paulista (HCFMB-Unesp, Botucatu), Hospital do Servidor Público Estadual de São Paulo (HSPE-SP, São Paulo), Instituto de Psiquiatria do Hospital das Clínicas da Faculdade de Medicina da Universidade de São Paulo (IP-FMUSP, São Paulo), Instituto de Psiquiatria da Universidade Federal do Rio de Janeiro (IP-UFRJ, Rio de Janeiro), Casa de Saúde Ana Nery (Salvador), Hospital das Clínicas da Faculdade de Medicina da Universidade Federal do Rio Grande do Sul (UFRGS, Porto Alegre) e Faculdade de Ciências Médicas da Santa Casa de São Paulo (São Paulo).

\section{REFERÊNCIAS}

American Psychiatric Asociation. Diagnostic and Statistical Manual of Mental Disorders. $4^{\text {th }}$ edition (DSM-IV). Washington DC, American Psychiatric Press; 1994.

Appolinario JC, McElroy SL. Pharmacological approaches in the treatment of binge eating disorder. Current Drug Targets, 5: 301-7, 2004.

Buchberger R, Wagner W. Fluvoxamine: safety profile in extensive post-marketing surveillance. Pharmacopsychiatry, 35: 101-8, 2002. 
Curzon G. Transmitteramines in depression. Psychological Medicine, 12: 465-70, 1982

Dalery J, Honig A. Fluvoxamine versus fluoxetine in major depressive episode: a double-blind randomised comparion. Human Psychopharmacology Clin Exp, 18: 379-84, 2003.

Delgado PL, Miller HL, Salomon RM, Licinio J, Krystal JH, Moreno FA, Heninger GR, Charney DS. Tryptophan-depletion challenge in depresssed patients treated with desipramine or fluoxetine: implications for the role of serotonin in the mechanism of antidepressant action. Biological Psychiatry, 46: 212-20, 1999.

Feighner JP, Boyer WF, Meredith CH, Hendrickson GG. A placebo-controlled inpatient comparison of fluvoxamine maleate and imipramine in major depression. International Clinical Psychopharmacology, 4: 239-44, 1989.

Gatti F, Bellini L, Gasperini M, Perez J, Zanardi R, Smeraldi E. Fluvoxamine alone in the treatment of delusional depression. American Journal of Psychiatry, 153: 414-6, 1996.

Haffmans PMJ, Timmerman L, Hoogduin CAL and the LUCIFER group. Efficacy and tolerability of citalopram with fluvoxamine in depressed outpatients: a double-blind, multicentre study. International Clinical Psychopharmacolog, 11: 157-64, 1996.

Lapin IP, Oxenkrug GF. Intensification of central serotonergic processes as a possible determinant of the thymoleptic action. Lancet, 18: 132-6, 1969.

Laws D, Ashford JJ, Anstee JA. A multicentre double-blind comparative trial of fluvoxamine versus lorazepam in mixed anxiety and depression treated in general practice. Acta Psychiatrica Scandinavica, 81: 185-9, 1990.

Montgomery SA. Antidepressant in long-term treatment. Annual Review of Medicine, 45: 447-57, 1994.

Mundo E, Rouillon F, Figueira ML, Stigler M. Fluvoxamine in obsessive-compulsive disorder: similar efficacy tolerability in comparison with clomipramine. Human Psychopharmacology Clin Exp,16: 461-8, 2001.

Nemeroff CB, Ninan PT, Ballenger J, Lydiard RB, Feighner J, Patterson WM, Greist J. Double-blind multicenter comparison of fluvoxamine versus sertraline in the treatment of depressed outpatients. Depression, 3: 163-9, 1995.

Nonogaki K, Nozue K, Takahashi Y, Yamashita N, Hiraoka S, Kumano H, Kuboki, Oka Y. Fluvoxamine, a selectivereuptake inhibitor, and $5 \mathrm{HT} 2 \mathrm{C}$ receptor inactivation induce appetite- suppressing effects in mice via 5-HT1B receptors. International Journal of Neuropsychopharmacology, 7: 1-7, 2006.

Ottevanger EA. The efficacy of fluvoxamine in patients with severe depression. British Journal of Clinical Research, 2: 125-32, 1991.

Rapaport M, Coccaro E, Sheline Y, Perse T, Holland P, Fabre L, Bradford D. A comparison of fluvoxamine and fluoxetine in the treatment of major depression. Journal of Clinical Psychopharmacology, 16: 373-8, 1996.

Sonawalla SB, Spillman MK, Kolsky AR, Alpert JE, Nierenberg AA, Rosenbaum JF, Fava M. Efficacy of fluvoxamine in the treatment of major depression with comorbid anxiety disorders. Journal of Clinical Psychiatry, 60: 580-3, 1999.

Stahl SM. Antidepressant treatment of psychotic major depression: potencial role of the sigma receptor. CNS Spectrums, 10: 319-23, 2005.

Thase ME, Haight BR, Richard N, Rockett CB, Mitton M, Modell JG, VanMeter S, Harnet AE, Wang Y. Remission rates following therapy with bupropion or selective serotonin reuptake inhibitors: a meta-analysis of original data from 7 randomized controlled trials. Journal of Clinical Psychiatry, 66: 974-81, 2005.

Westenberg HG, Sandner C. Tolerability and safety of fluvoxamine and other antidepressants. International Journal of Clinical Practice 60: 482-91, 2006.

Zanardi R, Franchini L, Gasperini M, Lucca A, Smeraldi A, Perez J. Faster onset of action in combination with pindolol in the treatment od delusional depression: a controlled study. Journal of Clinical Psychopharmacology, 18: 441-6, 1998.

Zanardi R, Franchini L, Serretti A, Perez J, Smeraldi E. Venlafaxine vs fluvoxamine in ter treatment of delusional depression: a pilot double-blind controlled study. Journal of Clinical Psychiatry, 61: 26-9, 2000.

Zohar J, Keegstra H, Barrelet L. Fluvoxamine as affective as clomipramine against symptoms of severe depression: results from a multicentre, double-blind study. Human Psychopharmacology Clin Exp, 18: 113-9, 2003. 